\title{
Validity and Reliability Examination of the Persian Version of the Iowa Satisfaction with Anesthesia Scale: The Result of Patient Satisfaction with Pain
}

\author{
Fatemeh Tarahomi $^{1}$, Saman Khadem ${ }^{1}$, Arezou Farajpour ${ }^{2}$, Hamed Beizaie ${ }^{3 *}$ \\ ${ }^{1}$ Faculty of Medicine, Mashhad Branch, Islamic Azad University, Mashhad, Iran \\ ${ }^{2}$ Faculty of Medicine, Shahid Beheshti University of Medical Sciences, Tehran, Iran \\ ${ }^{3}$ Department of Anesthesia, Faculty of Medicine, Mashhad Branch, Islamic Azad University, Mashhad, Iran
}

Article Info:

Received: 14 Apr 2018

Revised: 7 Jun 2018

Accepted: 10 Jun 2018

\section{A BSTRACT}

Introduction: Patient satisfaction is one of the important indicators in the quality of health care. Iowa Satisfaction with Anesthesia Scale (ISAS) questionnaire is one of the robust ques tionnaires measuring patient satisfaction in anesthesia care. However, the Persian version of this questionnaire is not available. Therefore, this study examines the validity and reliability of the Persian version of the ISAS questionnaire. This questionnaire has 11 questions ( 3 ques tions about pain, 6 questions about patient experiences during the surgery, and 2 questions about satisfaction of patient). Patient pain is one of the most important factors that modulate the patient satisfaction in anesthetic care. Materials and Methods: In this clinical trial, 230 candidates for eye surgery with topical anesthesia entered the study. After obtaining informed consent, the Persian version of the ISAS questionnaire was completed during and one hour after the operation. Reliability, face, content, concurrent and criterion validity was evaluated. Results: The questionnaire was completed for 205 patients. The mean age was $68.26 \pm 10.61$, including 98 (47.8\%) men and 107 (52.2\%) women. The average ISAS scores of patients are $1.02 \pm 0.65$ and its range was $-1.55-2.00$. Cronbach's alpha coefficient was 0.71 and there was a positive and significant correlation between test-retest scores. Facial and content validity was approved. In addition, a significant correlation with the satisfaction of the surgeon and visual analog scale (VAS) scores was observed, which suggests concurrent and criterion validity. In this study the satisfaction of pain extensively was considered and compared with VAS. There was significant correlation between the ISAS questionnaire and VAS ( $r=-0.524, p=0.0001)]$. Conclusion: The findings of this study confirm the validity

Key words:

1. Anesthesia

2. Patient Satisfaction 3. Pain

*Corresponding Author: Hamed Beizaie

E-mail:Hmd_beyzaii@yahoo.com 


\title{
بررسى روايى و يايايى نسخهُ فارسى سنجش رضايتمندى بيماران از مراقبت هاى بيهوشى Iowa: نتيجئ رضايتمندى بيمار ان بان درد بينمان إن
}

\author{
فاطمه ترحمى'، سامان خادم'، آرزو فرج بور '، حامد بيضايى" \\ 'دانشكده يزشكى، واحد مشهد، دانشكاه آزاد اسلامى، مشهد، ايران \\ 'دانشكده يزشكى، دانشكاه علوم يز شكى شهيد بهشتى، تهر ان، ايران

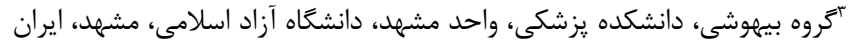

اطلاعات مقاله:

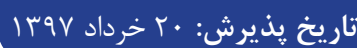

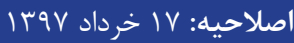

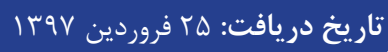

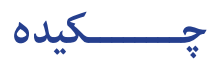

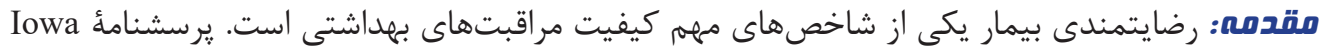

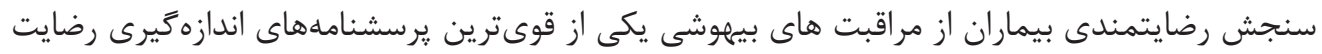

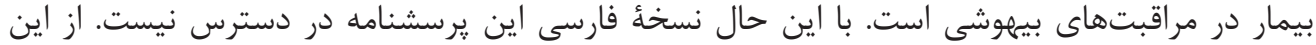

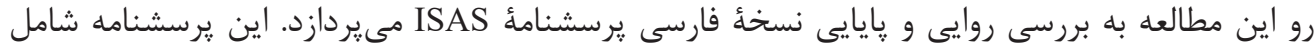

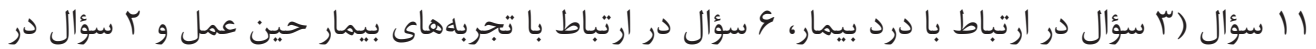

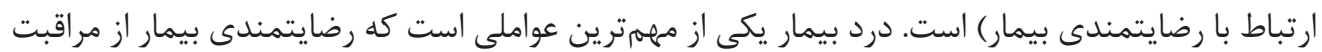

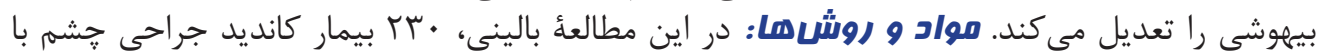

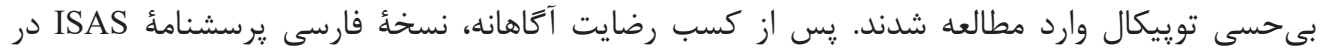

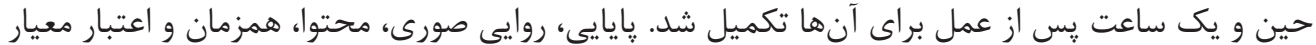

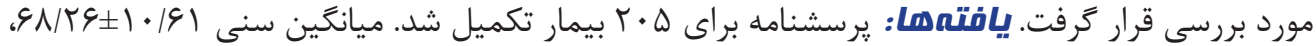

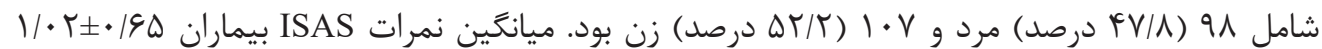

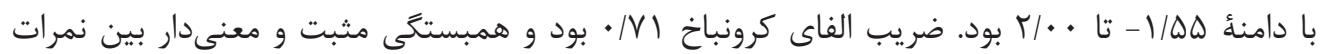

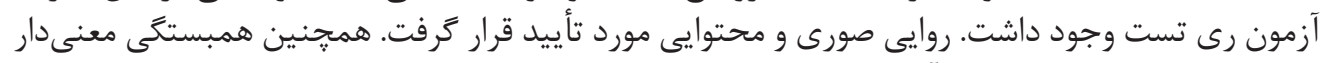

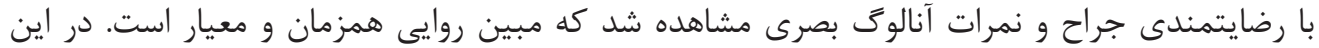

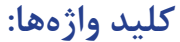

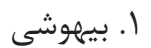
r. رائمايتدى بيمار r. r. درض

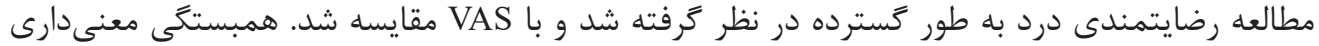

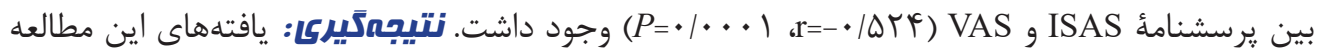

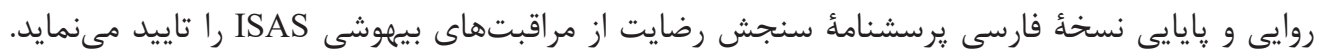

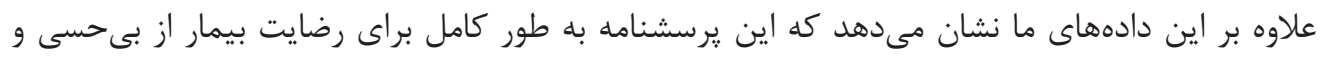
درد مفيد است. 
بــه بسـترى شـدن بيمـار نيست امــا ايسـن روش نيـز درد نســــاً

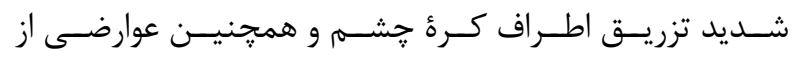

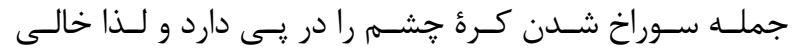

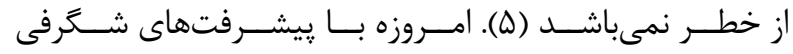

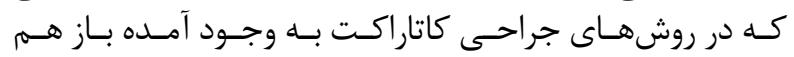

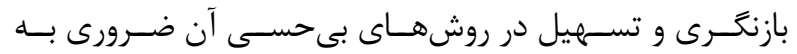

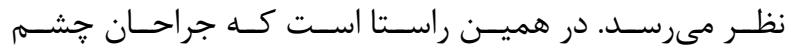

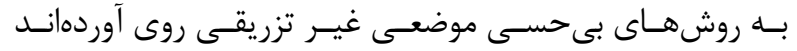

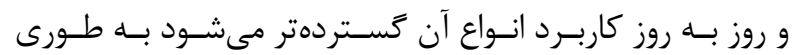

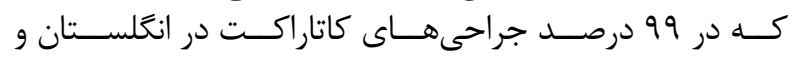

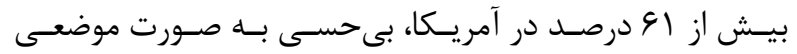

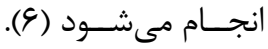

در حقيقـت روش بى حسـى تويــكال روشـى ارزشـمند اسـت

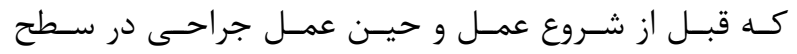

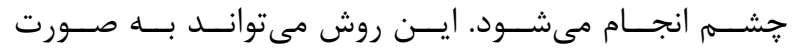

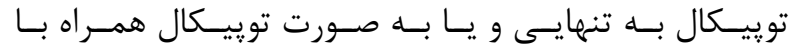

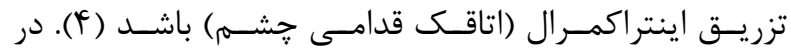

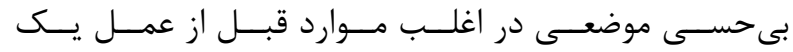

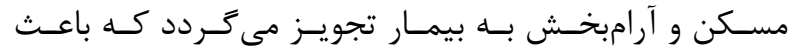

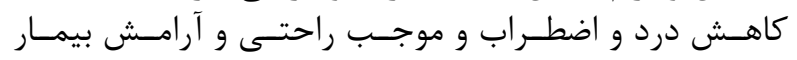

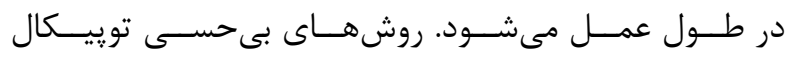

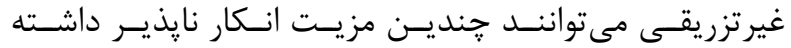

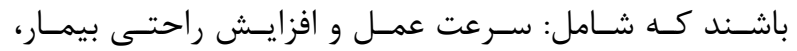

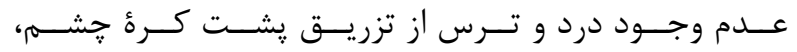

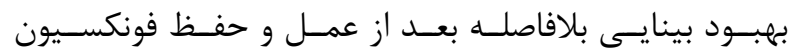

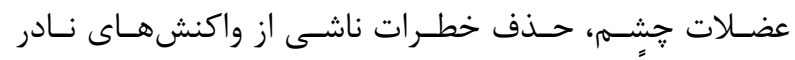

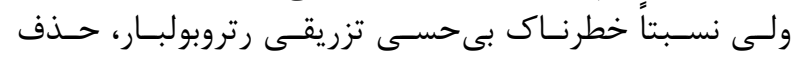

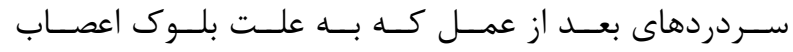

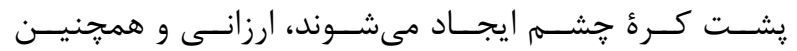

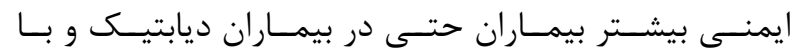

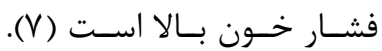

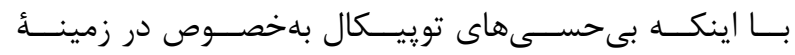

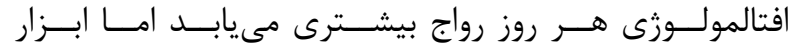

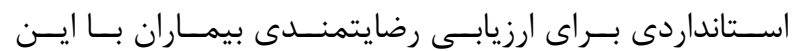

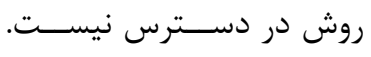

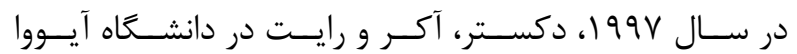

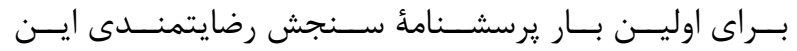

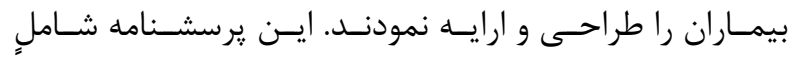

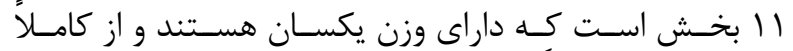

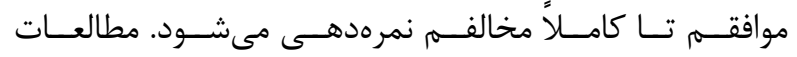

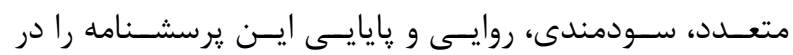

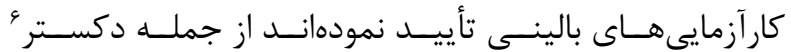

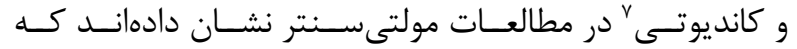

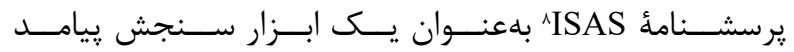

${ }^{1}$ Klok

${ }^{2}$ Iowa

${ }^{3}$ Neurorasptors

${ }^{4}$ Neurotransmitter
مقلهمه

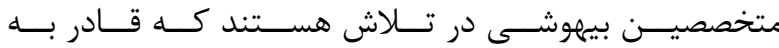

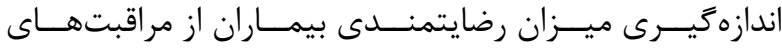

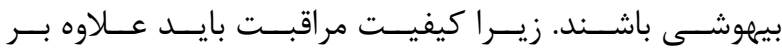

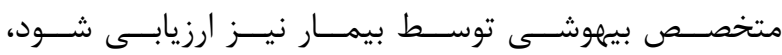

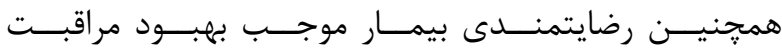

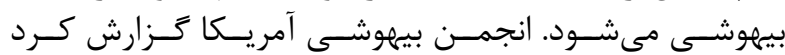

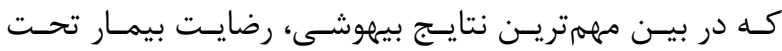

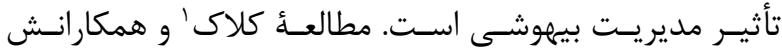

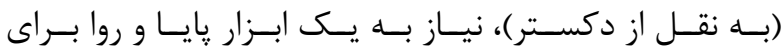

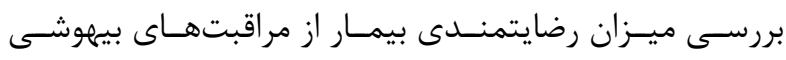

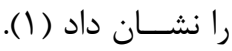

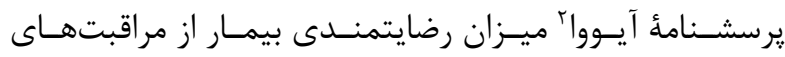

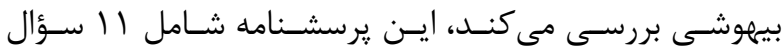

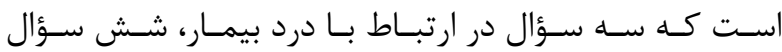

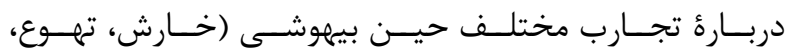

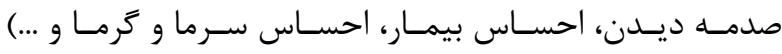

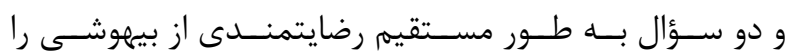

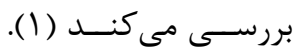

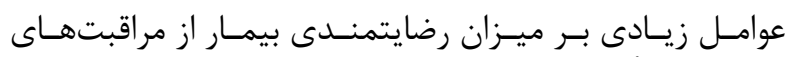

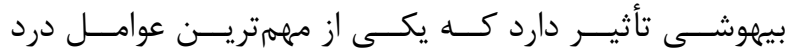

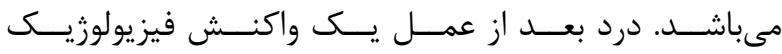

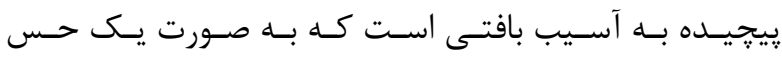

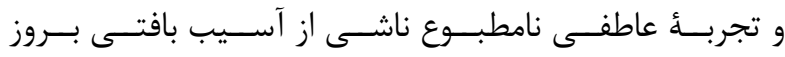

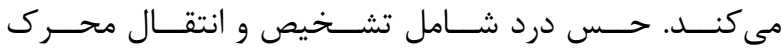

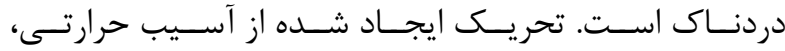

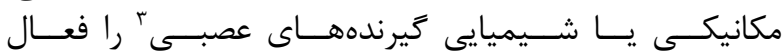

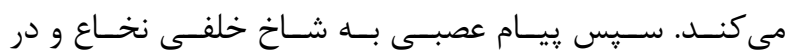

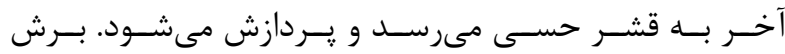

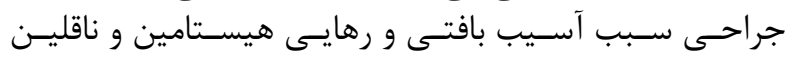

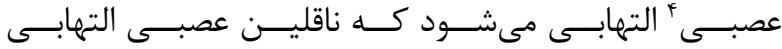

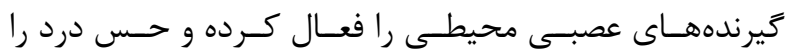

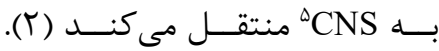

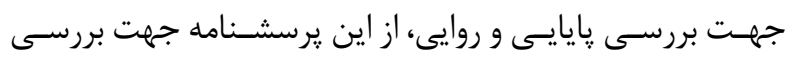

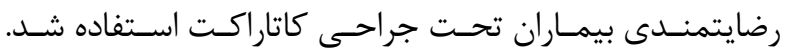

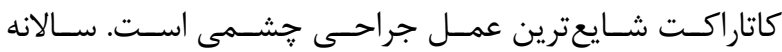

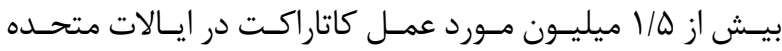

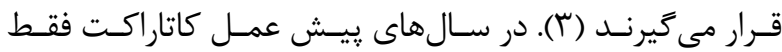

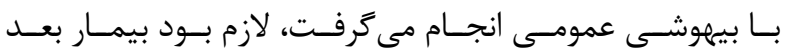

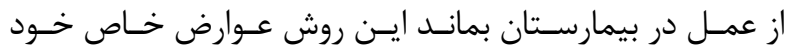

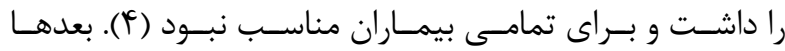

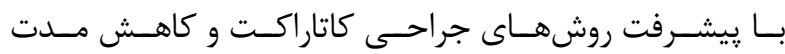

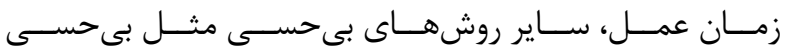

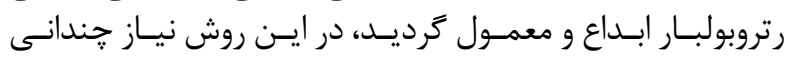

\footnotetext{
${ }^{5}$ Central nervous system

${ }^{6}$ Dexter

${ }^{7}$ Candiotti

${ }^{8}$ Iowa satisfaction with anesthesia scale
} 


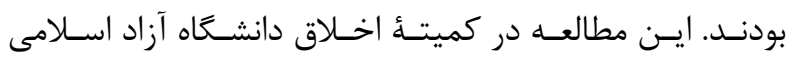

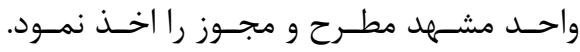

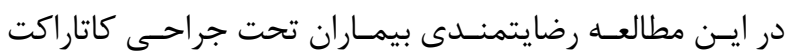

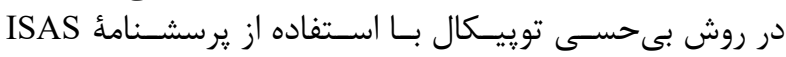

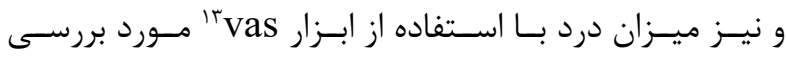

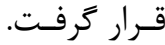

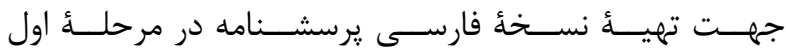

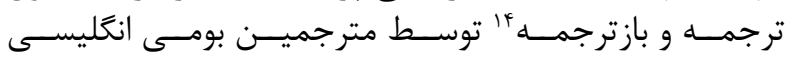

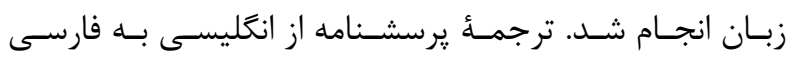

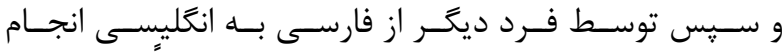

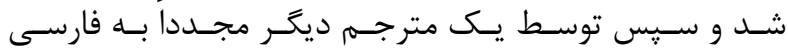

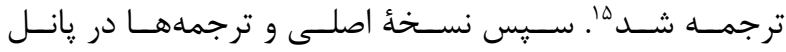

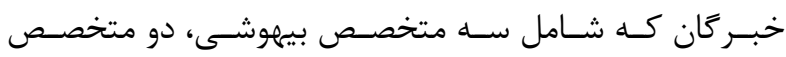

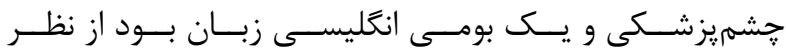

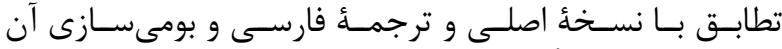

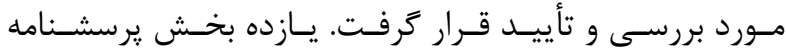

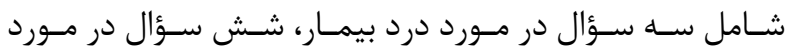

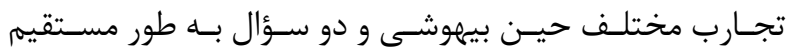

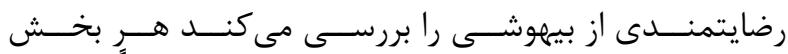

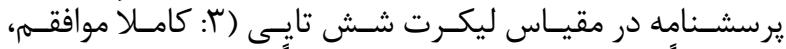

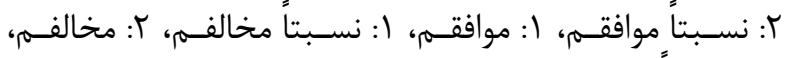

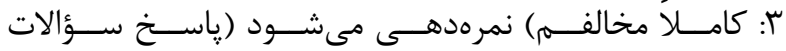

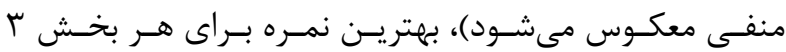

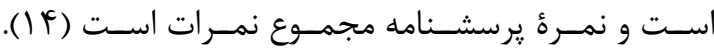

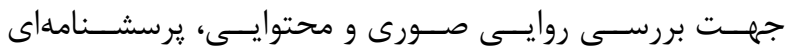

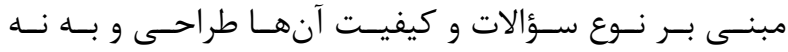

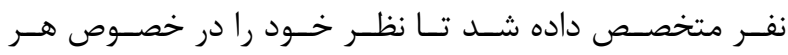

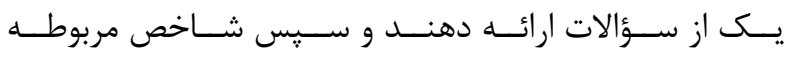

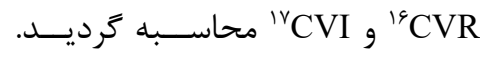

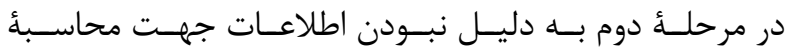

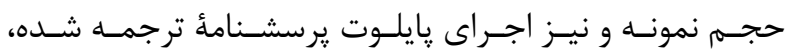

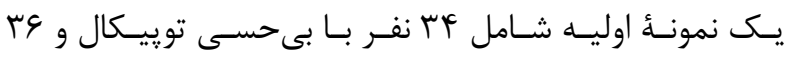

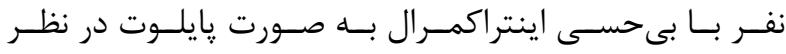

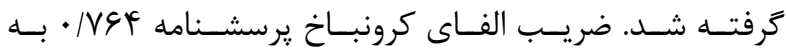

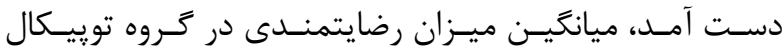

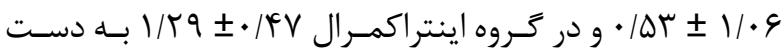

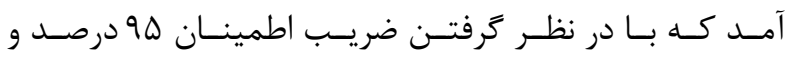

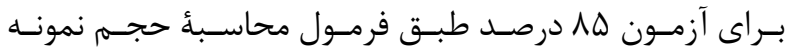

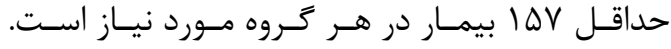

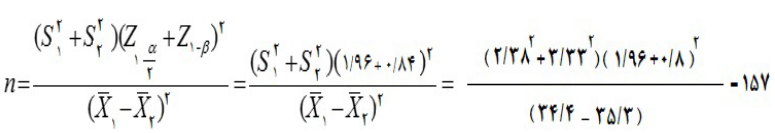

\footnotetext{
${ }^{9}$ Procedure

${ }^{10}$ Dexmedetomidine

${ }^{11}$ Chanthog

${ }^{12}$ American society of anesthesiologists

${ }^{13}$ Visual analog scale
}

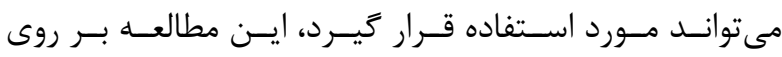

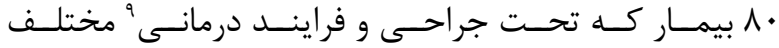

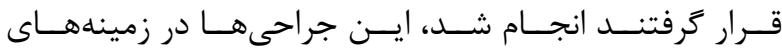

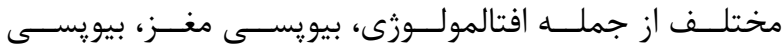

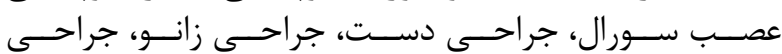

يلاسـتيك، زنيكولـــوزى و ... بودنـــــ (1).

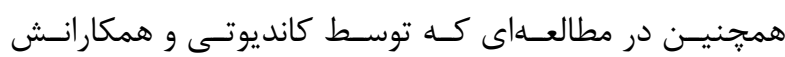

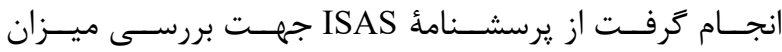

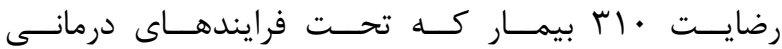

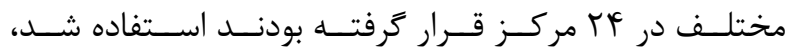

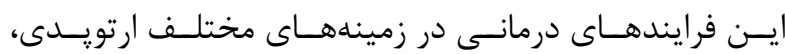

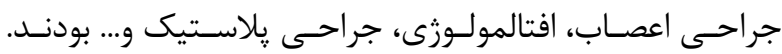

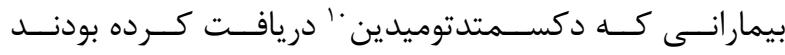

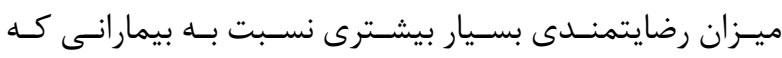

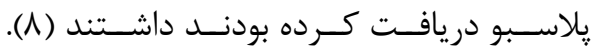

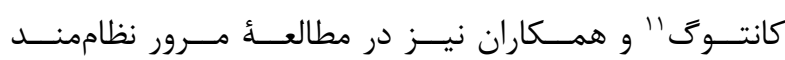

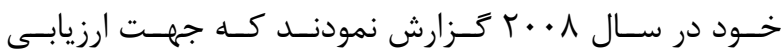

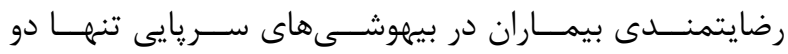

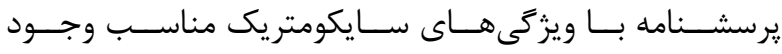

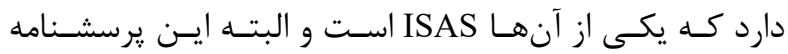

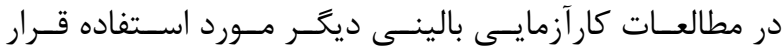

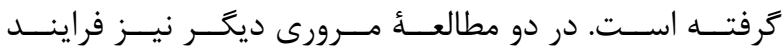

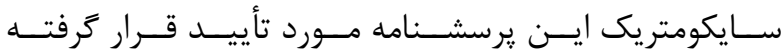

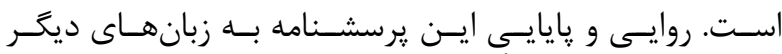

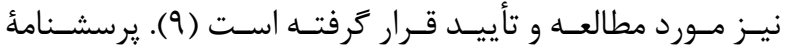

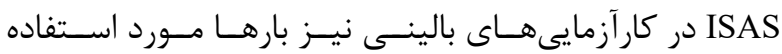

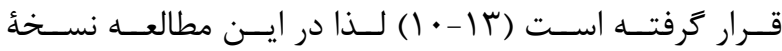

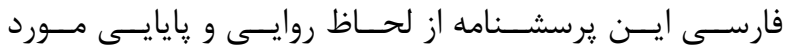

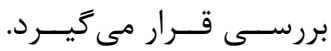

مواد و روشها

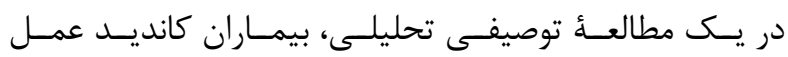

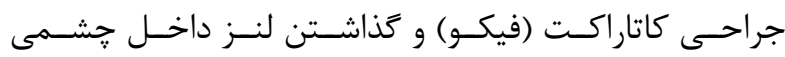

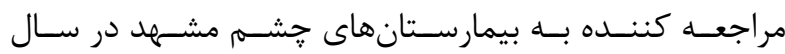

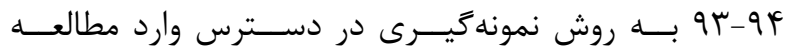

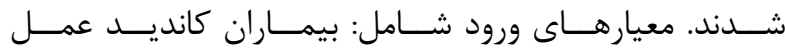

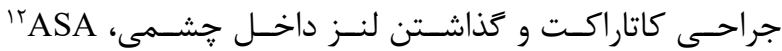

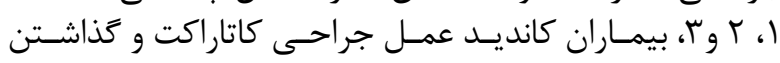

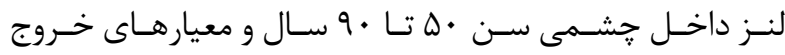

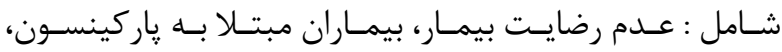

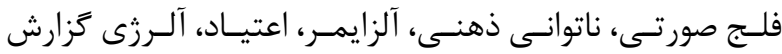

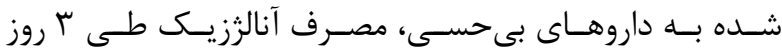

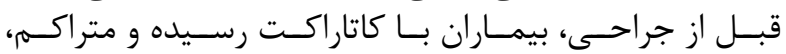

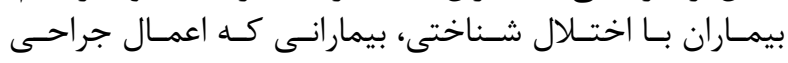

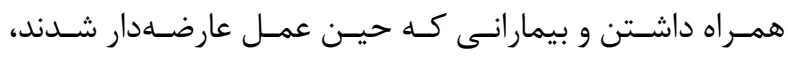

\footnotetext{
${ }^{14}$ Translation-retranslation

${ }^{15}$ Back-translation

${ }^{16}$ Content validity ratio

${ }^{17}$ Content validity index
} 


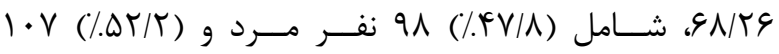

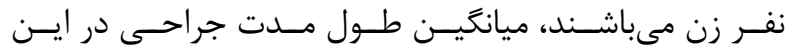

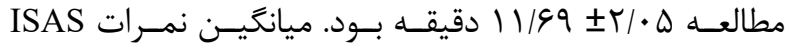

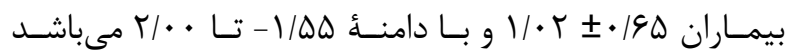

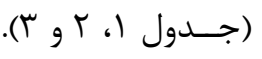

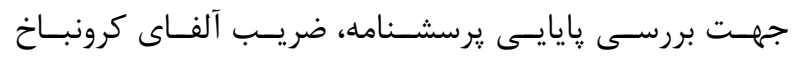

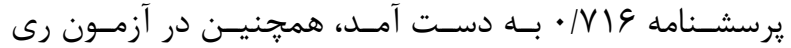

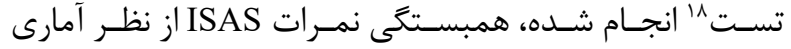

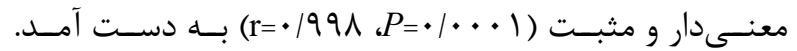

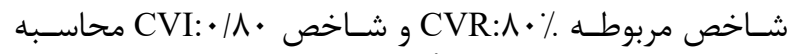

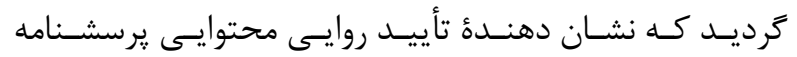

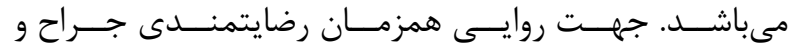

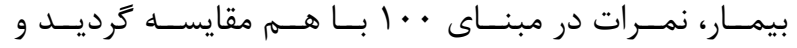

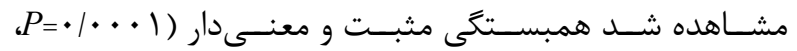

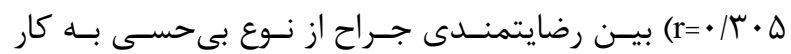

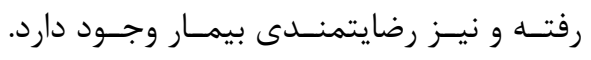

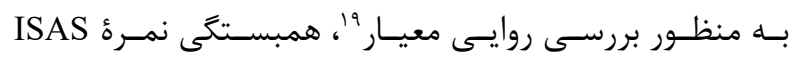

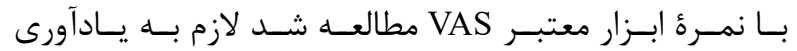

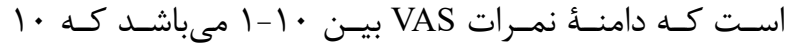

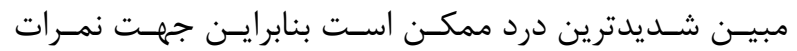

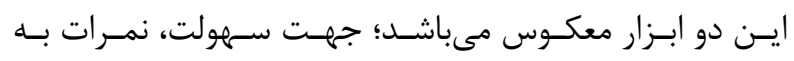

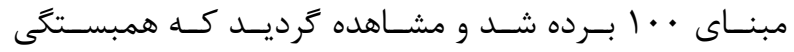

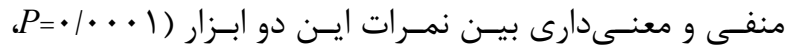
(r=-•/DTF

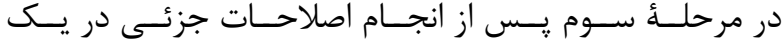

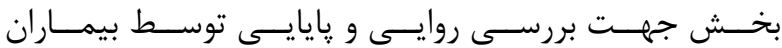

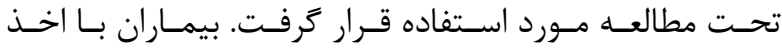

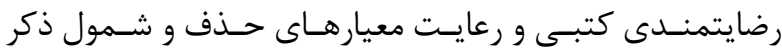

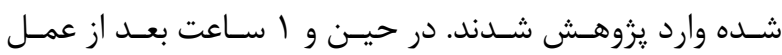

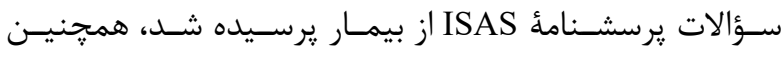

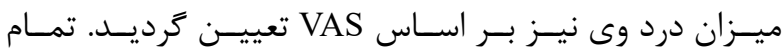

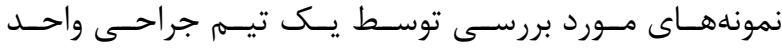

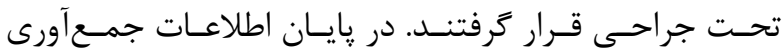

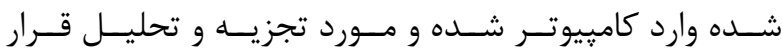

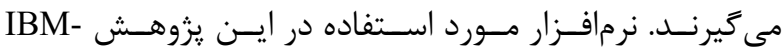

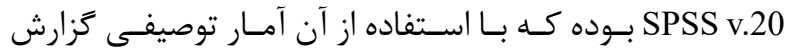

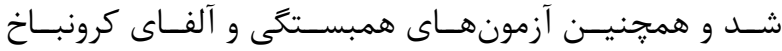

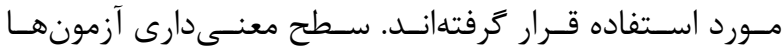

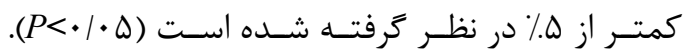

بافتهها

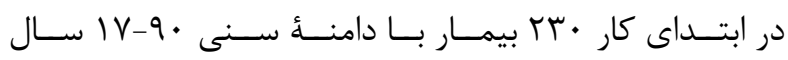

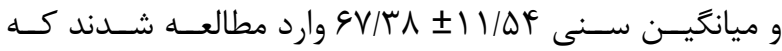

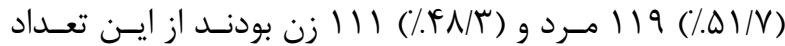

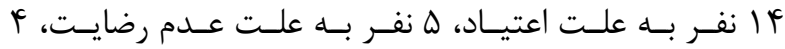

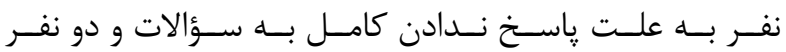

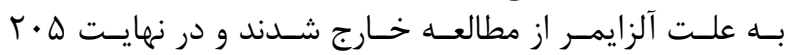

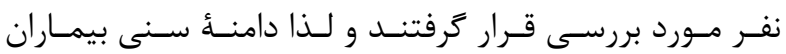

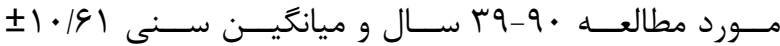

جدول ا- توزيع جنسيت بيماران شركتكننده در مطالعه.

\begin{tabular}{|c|c|c|c|c|c|c|}
\hline سطح معنى دارى & انحر اف معيار & ميانحين & טرص & تعداد & جنس & \\
\hline$P<\cdot 1 \cdot \Delta$ & f/VT & $\Delta 1 / V$ & FV/A & 9人 & مرد & متغير \\
\hline$P<\cdot 1 \cdot \Delta$ & F/FF & $r \cdot / r$ & $\Delta T / r$ & $1 \cdot v$ & 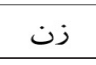 & \\
\hline
\end{tabular}

جدول r - توزيع نمرة ISAS بيماران شركت كننده در مطالعه.

\begin{tabular}{|c|c|c|c|c|c|}
\hline سملح معنى دارى & انحراف معيار & ميانخين & بيشترين & كمترين & متغير \\
\hline$P<\cdot 1 \cdot \omega$ & $1 \cdot|9|$ & ૬人/T૬ & 9 . & 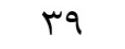 & سن \\
\hline$P=\cdot 1 \cdots+1$ & $\Delta / \uparrow q$ & F人/1 $q$ & $\Delta V$ & r - & نمره \\
\hline
\end{tabular}

جدول ب- توزيع مدت زمان عمل (بر حسب دقيقه) در بيماران شركت كننده در مطالعه.

\begin{tabular}{|c|c|c|c|}
\hline سطح معنى دارى & انحراف معيار & ميانگين & متغير \\
\hline$P<\cdot 1 \cdot \Delta$ & $r / \cdot \Delta$ & $11 / 99$ & بت عمل \\
\hline
\end{tabular}

${ }^{18}$ Test-retest

${ }^{19}$ Criterion validity

${ }^{20}$ Fung 


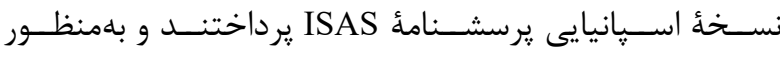

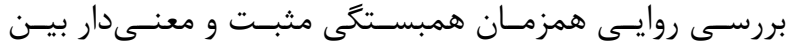

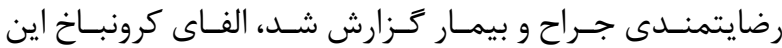

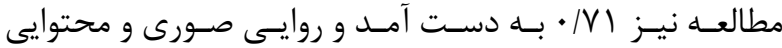

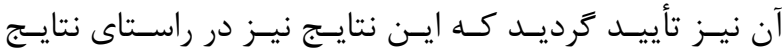

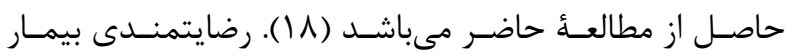

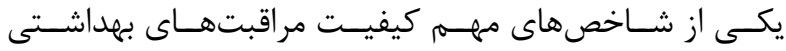

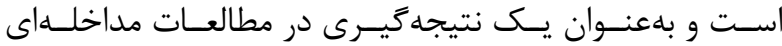

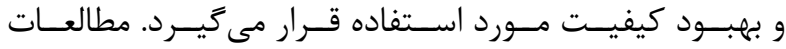

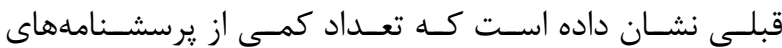

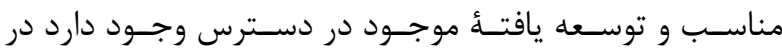

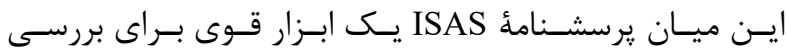

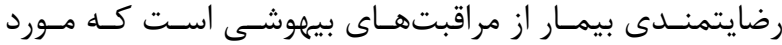
قبـول بيمـاران و يزشـكان اســـ (19).

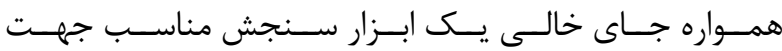

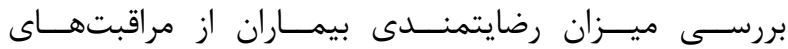

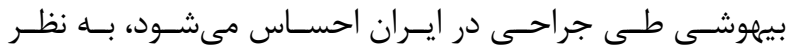

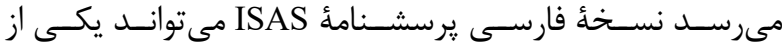

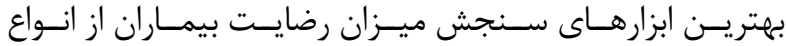

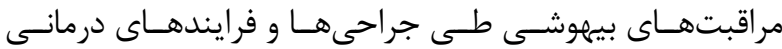

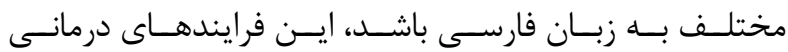

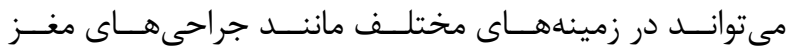

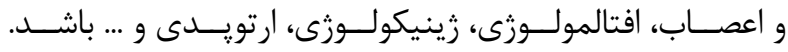

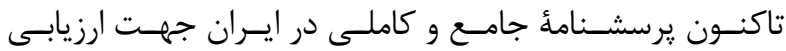

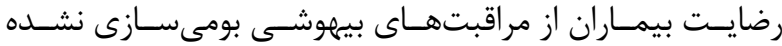

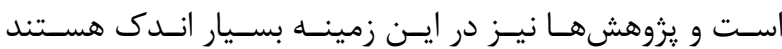

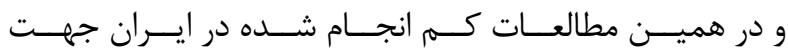

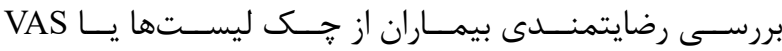

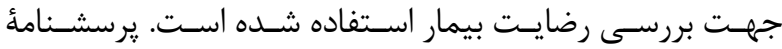

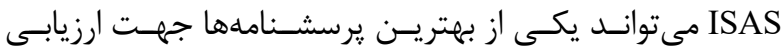

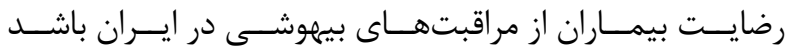

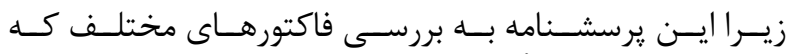

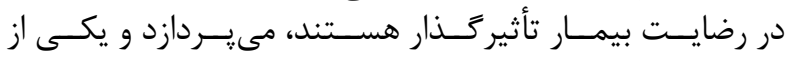

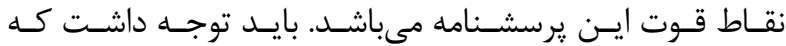

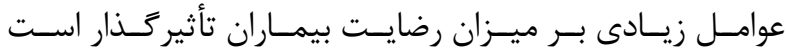

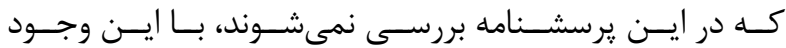

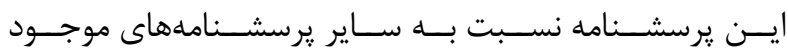

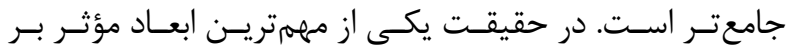

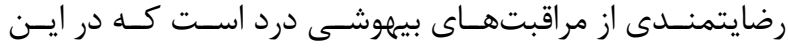

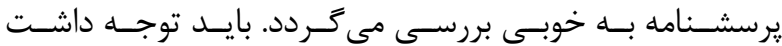

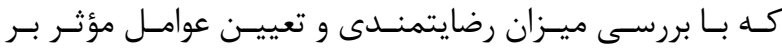

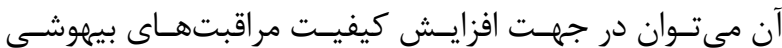
و كاهـش نارضايتـى بيمـار كوشـيد.

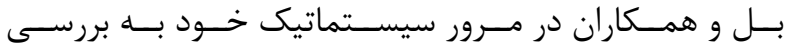

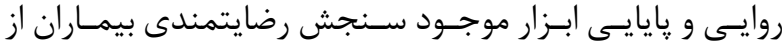

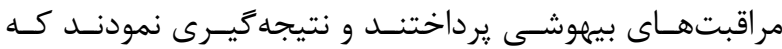

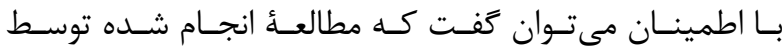

بحث و نتيجلهَيرى

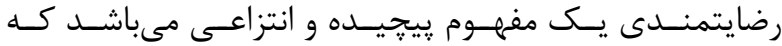

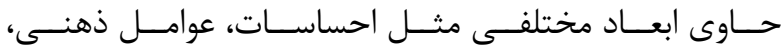

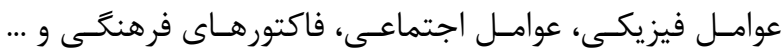

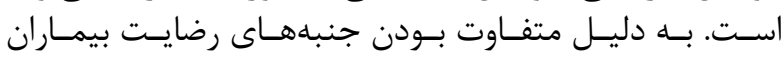

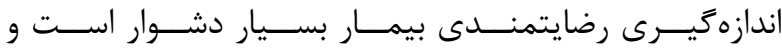

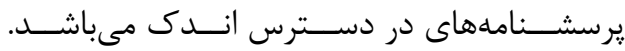

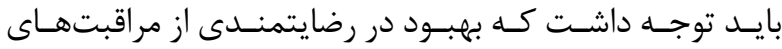

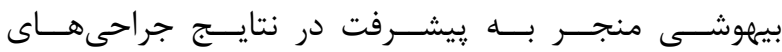

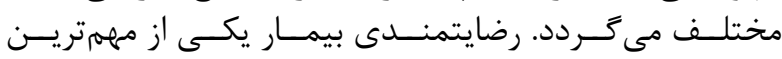

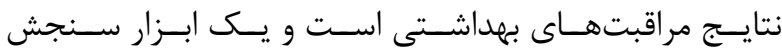

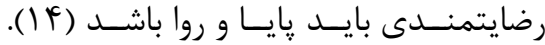

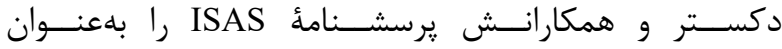

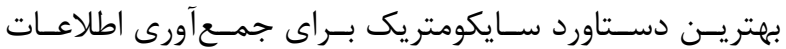

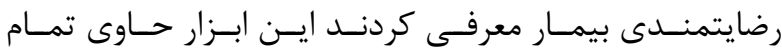

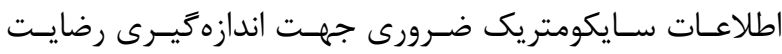

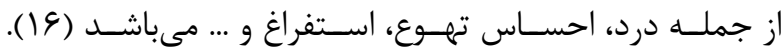

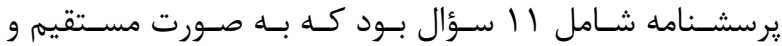

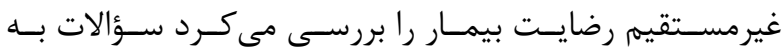

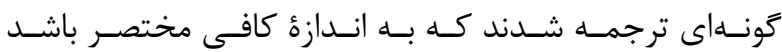

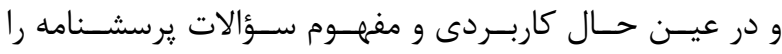

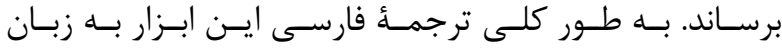

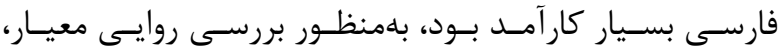

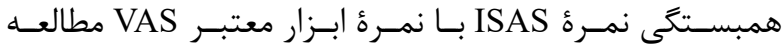

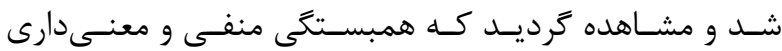

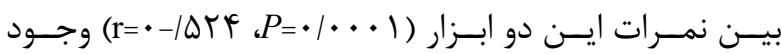

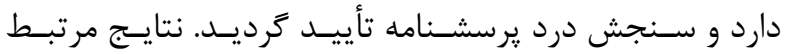

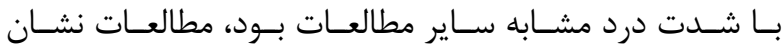

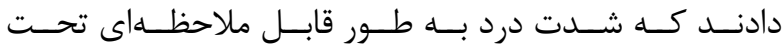

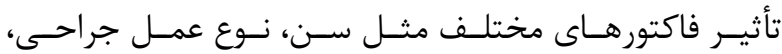

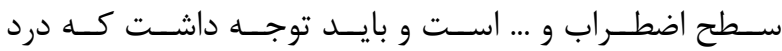

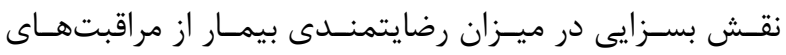

بيهوشـى دارد (IV) (IV)

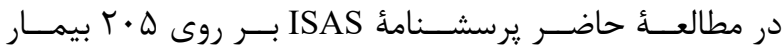

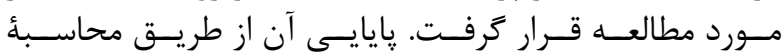

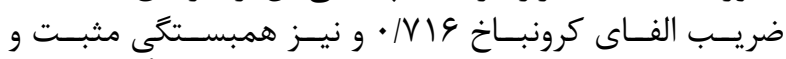

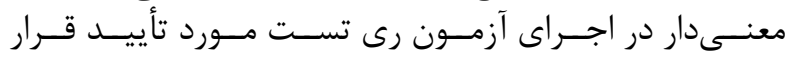

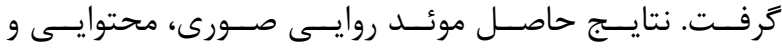

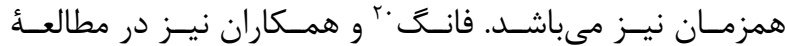

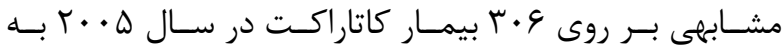

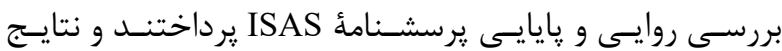

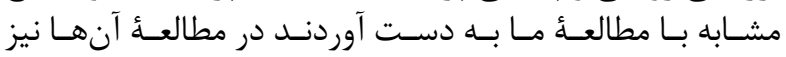

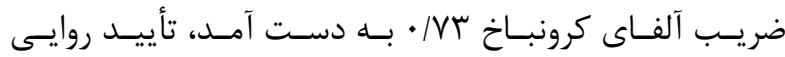

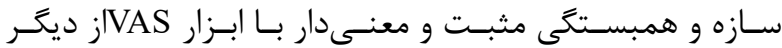

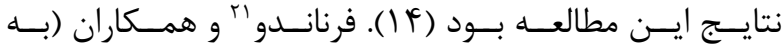

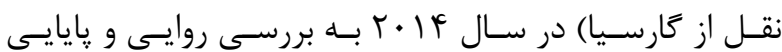




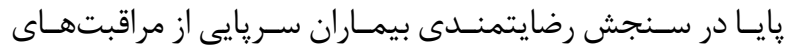

$$
\begin{aligned}
& \text { بيهوشـى تأييـد مىنمايسـد. }
\end{aligned}
$$

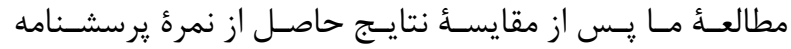

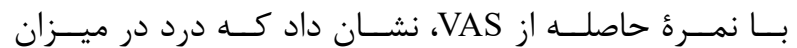

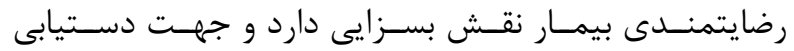

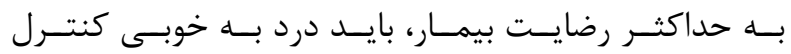

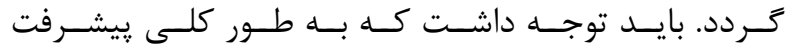

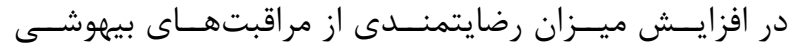

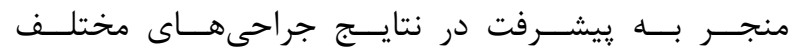

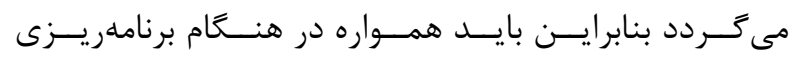

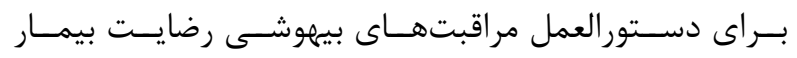

$$
\begin{aligned}
& \text { در نظــر كرفتـهـ شـود. }
\end{aligned}
$$

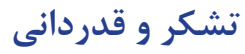

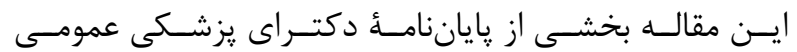

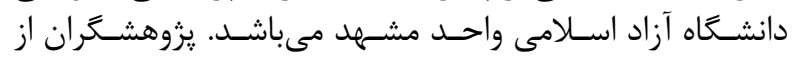

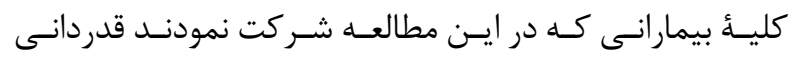

$$
\begin{aligned}
& \text { مىنماينــد. }
\end{aligned}
$$

1. Dexter F, Candiotti KA. Multicenter assessment of the Iowa Satisfaction with anesthesia scale, an instrument that measures patient satisfaction with monitored anesthesia care. Anesth Analg. 2011; 113(2): 364-8.

2. Pardo Jr. Local anesthesia. Miller Ronald D, Manuel C, Pardo Jr. Basic of Anesthesia. $6^{\text {th }}$ ed. California San Francisco: Elsevier; 2011. P. 740-1.

3. Katz J, Feldman MA, Bass EB, Lubomski LH, Tielsch JM, Petty BG, et al. Injectable versus topical anesthesia for cataract surgery: patient perceptions of pain and side effects. the study of medical testing for cataract surgery study team. Ophthalmology. 2000; 107(11): 2054-60.

4. Leaming DV. Practice styles and preferences of ASCRS members-2003 survey. J Cataract Refract Surg. 2004; 30(4): 892-900.

5. Amiraslanzadeh Gh GD, Parish M, Nourinia R, Sedaghat K. Evaluation of effectiveness and safety of lidocaine gel as anesthetic. M J Tabriz University of Medical Sciences. 2008; 30(2): 25-8.

6. Johnston RL, Sparrow JM, Canning CR, Tole D, Price NC. Pilot national electronic cataract surgery survey: i. method, descriptive, and process features. Eye (Lond). 2005; 19(7): 788-94.

7. Nwosu SN, Nwosu VO, Anajekwu C, Ezenwa A. Retrobulbar versus subconjunctival anesthesia for cataract surgery. Niger J Clin Pract. 2011; 14(3): 280-3.

8. Candiotti KA, Bergese SD, Bokesch PM, Feldman MA,

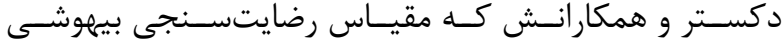

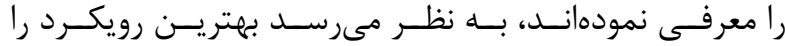

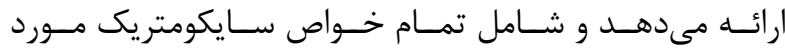

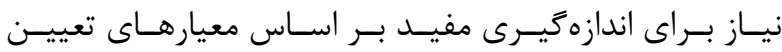

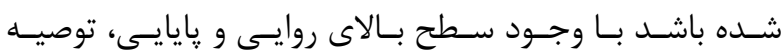

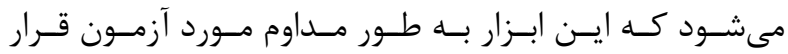

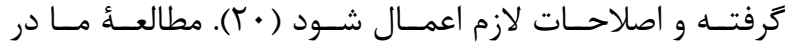

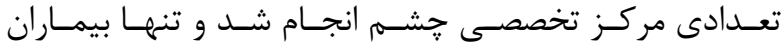

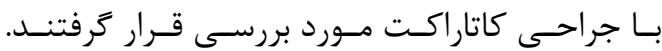

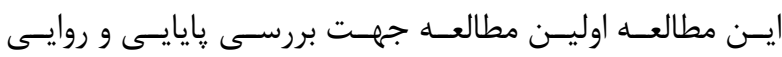

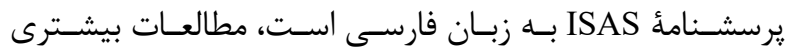

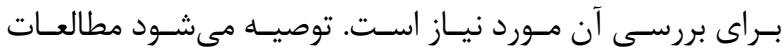

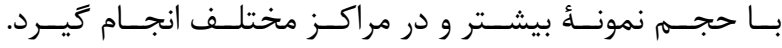

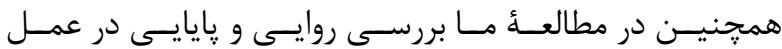

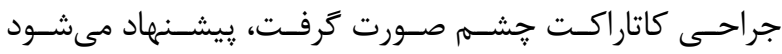

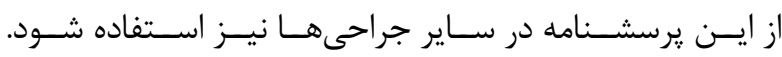

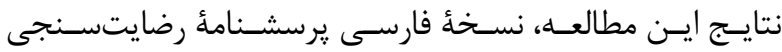

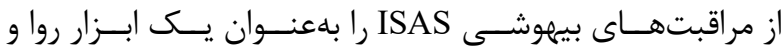

منابع

Wisemandle W, Bekker AY, et al. Monitored anesthesia care with dexmedetomidine: a prospective, randomized, double-blind, multicenter trial. Anesth Analg. 2010; 110: 47-56.

9. Chanthong P, Abrishami A, Wong J, Chung F. Patient satisfaction in ambulatory anesthesia: a systamatic review. Canadian J Anesthesia. 2008; 55: 4715771-2.

10. Lee EJ, Khandwala M, Jones CA. A randomised controlled trial to compare patient satisfaction with two different types of local anaesthesia in ptosis surgery. Orbit. 2009; 28(6): 388-91.

11. Ahmad N, Zahoor A, Motowa SA, Jastaneiah S, Riad W. Satisfaction level with topical versus peribulbar anesthesia experienced by same patient for phacoemulsification. Saudi J Anaesthesia. 2012; 6(4): 363-6.

12. Masood N, Saleem MA, Masroor R, Uddin S. Evaluation patient's satisfaction of anesthesia care. Pakistan J MED. 2008; 2(5): 41.

13. Chan WH, Biswas S, Lloyd IC, Wraith E, Jones S, Mercer J, et al. What makes a good operation great? factors determining patient satisfaction with local anaes thesia in cataract surgery. J Eye. 2013; 27(9): 1112-4.

14. Fung D, Cohen M, Stewart S, Davies A. Can the iowa satisfaction with anesthesia scale be used to measure patient satisfaction with cataract care under topical local anesthesia and monitored sedation at a community hospital? Anesth Analg. 2005; 100(6): 1637-43. 
15. Baroudi D, Nofal W. Patient safety in anes thesia. Internet $\quad \mathrm{J} \quad$ Health. 2009; 8(2): 124-43.

16. Dexter F, Aker J, Wright WA. Development of a measure of patient satisfaction with monitored anes thesia care: the iowa satisfaction with anesthesia scale. Anesthesiology. 1997; 87(4): 856-64.

17. Kalkman CJ, Visser K, Moen J, Bonsel GJ, Grobbee DE, Moons KGM. Preoperative prediction of severe pos toperative pain Pain. 2003; 105(3): 415-23.

18. García LFJ, Capera ADR. Validation to spanish of the iowa satisfaction with anesthesia scale (isas) for monitored anesthesia care in ophthalmic surgery. Colombian Journal of Anesthesiology. 2014; 42(4): 272-80.

19. Barnett SF, Alagar RK, Grocott MP, Giannaris S, Dick JR, MoonesingheSR. Patient-satisfaction measures in anesthesia qualitative: systematic review. Anesthesiology. 2013; 119(2): 452-78.

20. Bell DM, Halliburton JR, Preston JC. An evaluation of anesthesia patient satisfaction instruments. AANA J. 2004; 72: 211-7. 